\title{
e-Phaïstos
}

e-Phaïstos

Revue d'histoire des techniques / Journal of the history

of technology

IX-1 | 2021

Autour de Léonard de Vinci

\section{Concevoir et faire : économie et technologie à l'époque moderne}

Entretien avec Hélène Vérin

Designing and Doing. Economy and Technology in Modern Times. An interview with Hélène Vérin

\section{Hélène Vérin}

\section{OpenEdition}

Journals

Édition électronique

URL : https://journals.openedition.org/ephaistos/8634

DOI : 10.4000/ephaistos.8634

ISSN : 2552-0741

Éditeur

IHMC - Institut d'histoire moderne et contemporaine (UMR 8066)

\section{Référence électronique}

Hélène Vérin, «Concevoir et faire : économie et technologie à l'époque moderne », e-Phaïstos [En ligne], IX-1 | 2021, mis en ligne le 27 avril 2021, consulté le 19 mars 2022. URL : http://

journals.openedition.org/ephaistos/8634; DOI : https://doi.org/10.4000/ephaistos.8634

Ce document a été généré automatiquement le 19 mars 2022.

Tous droits réservés 


\title{
Concevoir et faire : économie et technologie à l'époque moderne
}

\author{
Entretien avec Hélène Vérin \\ Designing and Doing. Economy and Technology in Modern Times. An interview \\ with Hélène Vérin
}

Hélène Vérin

\begin{abstract}
Chère Hélène, tu as publié trois ouvrages majeurs, l'un sur la manière dont l'entreprise a été pensée, l'autre sur l'intelligence technique, le troisième sur la réduction en art, une méthode pour penser l'action qui a pris forme dans l'Europe de l'époque moderne. Ces trois domaines sont différents, du moins en apparence. II me semble néanmoins que chacun d'eux analyse sous un angle différent, la "pensée de l'action», son émergence, ou plus exactement la manière dont elle s'est objectivée et structurée à l'époque moderne.
\end{abstract}

Si l'on exclut ma passion de jeunesse pour Goethe et son « au commencement était l'action ${ }^{1}$ ", l'idée de travailler sur la pensée de l'action m'est venue durant la préparation de ma licence de philosophie, en 1965-68, avec la lecture de Husserl. Mais si l'on veut faire date, alors c'est plus précisément durant un cours de philosophie générale de Yvon Belaval ${ }^{2}$, le seul sans doute de nos professeurs de la faculté des lettres de la Sorbonne qui s'intéressait à la question, que j'ai compris que j'allais travailler sur ce qu'était concevoir, penser pour agir, ce qu'était la pensée pour l'agir. Et c'est bien ce que j'ai continué à faire jusqu'à présent. D'emblée, cela me plaçait à un certain niveau d'abstraction favorable à l'étude d'une multiplicité de domaines d'exercice et de modalités d'exercice de l'activité humaine.

\section{Entreprise, ingénieur, réduire en art : une histoire des manières de concevoir}

Ceci dit, pour comprendre pourquoi j'ai orienté mon travail vers l'histoire, je dois dire que je supportais mal la lecture désincarnée de l'activité philosophique, que proposaient traditionnellement les philosophes où, dans le meilleur des cas, on règle -et surtout on réglait- la question de son historicité en un seul paragraphe, par la 
référence à un "contexte historique » qui se contentait d'évacuer la question. Or, l'insertion de toute pensée et méthode de penser dans une certaine histoire était pour moi une question majeure et certainement la plus passionnante, avec en retour, l'idée qu'il y avait une histoire des manières de concevoir qui méritait d'être explorée. Il ne faut pas oublier que tout cela se passait à un moment où la grande entreprise de Michel Foucault battait son plein, que le prestige des historiens était au zénith, et j'avoue que lorsque j'ai découvert Braudel, ce fut un émerveillement. Par ailleurs, je trouvais Foucault passionnant mais aussi beaucoup trop systématique. Il y avait place pour une approche plus respectueuse, plus en profondeur, de ces époques pour lesquelles il nous proposait ses hypothèses.

Pour revenir sur les trois thèmes : entreprise, ingénieur et réduire en art, l'un m'a conduit à l'autre et à chaque fois mon point de départ a été le mot, les mots et les jeux des idées et du sens dans les mots tels qu'ils se présentaient, se modifiaient, se transformaient, au fur et à mesure que j'entrais plus avant dans leur découverte. Mon principe était toujours le plus grand respect pour les valeurs et les significations qui affleuraient, s'imposaient ou s'effaçaient au profit d'autres encore dans les usages. Je pense que c'est ce qui m'a permis de mettre en lumière l'expression " réduire en art » et le phénomène culturel qu'elle a recouvert historiquement.

Ceci dit, ces trois thèmes portent sur la même époque historique, s'inscrivent dans le même essor de la science et du capitalisme marchand et se tiennent à l'articulation entre ces deux grandes déterminations de l'activité humaine. Quant à la science, ce qui ressort pour moi est que durant cette époque moderne, jusqu'au milieu du XVIII ${ }^{e}$ siècle, le plus marquant dans les transformations socio-techniques n'est pas tant l'essor théorique que le très progressif renouvellement des outils scientifiques effectivement disponibles dans les usages, et ainsi un contexte favorable à l'ouverture du domaine des arts - entre sciences et métiers.

Néanmoins, c'est dans le cadre de ton doctorat d'état en philosophie que tu as élaboré Entrepreneurs, entreprise..., ton premier ouvrage...

La philosophie a été ma première passion intellectuelle, dès que j'ai su que cela existait, quand j'avais seize ans. Il a été très compliqué et difficile de m'y adonner, vue la violence de l'irruption de l'Histoire dans mon histoire personnelle, au cœur de la guerre d'Algérie et de son indépendance. Autant dire que mes études, commencées à Alger, ont été chaotiques et entravées par toutes sortes de circonstances. Arrivée à Paris en 1965, j'ai repris mes études à la Sorbonne. Le sujet de ma maîtrise, proposé par Pierre Macherey ${ }^{3}$ en 1968 : Le statut de la critique dans Le Capital de Karl Marx, m'a orientée vers l'épistémologie et l'histoire des idées économiques. La proposition de Pierre Vilar ${ }^{4}$ de travailler sur les concepts d'entreprise et de profit venait à point. Il n'arrivait pas à trouver parmi ses étudiants historiens quelqu'un qui choisisse de vraiment s'y adonner. Ce fut moi, pour le concept d'entreprise. Mais très vite ce furent les notions d'entreprise et d'entrepreneur, les champs dans lesquels ces notions prenaient forme et sens, les temps et les espaces historiques de leurs transformations. Néanmoins, j'ai eu un mal fou à trouver un directeur parmi les professeurs de philosophie, et Jean-Toussaint Desanti ${ }^{5}$ qui a bien voulu accepter m'a averti qu'il ne pourrait en rien m'aider, n'y connaissant rien. Lorsque j'ai été voir Pierre Vilar, comme me le suggérait Desanti, il m'a reçu très fraîchement, m'assurant qu'il ne voulait plus avoir affaire avec des philosophes. Il était alors en conflit ouvert avec Foucault et ses partis-pris dans le choix de ses sources, insupportables à 
l'historien qu'il était. Heureusement après un an il a compris mon projet. Mais à vrai dire j'ai été totalement livrée à moi-même tant pour l'élaboration de ma thèse, que pour sa soutenance. Pour la soutenance, mon jury comprenait des philosophes, JeanToussaint Desanti, François Châtelet ${ }^{6}$, Yvon Belaval et Jean-Marie Vincent $^{7}$ et un historien, Pierre Vilar. C'était la confirmation par ces choix de Desanti, du caractère transdisciplinaire de mon approche.

Le choix que tu as fait d'analyser l'entreprise comme étant une notion, une idée, avant que de devenir une organisation dédiée à la production, dans le sens actuel du terme, vient-il de cette approche transdisciplinaire? Et, question annexe: comment expliques-tu que ces notions soient apparues beaucoup plus tardivement dans le monde anglophone?

On pourrait dire que c'est tout l'inverse. C'est d'avoir rencontré les notions d'entreprise et d'entrepreneur aux sens politique, juridique, économique, polémologique, que j'ai été conduite à étendre mes investigations dans ces multiples domaines. En quelque sorte, j'ai été guidée par mes découvertes. C'est vrai que ce n'était pas très académique. Jean-Toussaint Desanti m'a dit, qu'avec un tel sujet je ne ferai pas carrière, et il m'a précisé, avec l'humour qu'on lui a reconnu, qu'il fallait choisir un philosophe et si possible un sujet sur ce philosophe, comme "la mémoire chez Plotin ». Ce fut son exemple. Toutefois, l'idée de travailler sur l'entreprise et les entrepreneurs, sur les transformations et les constantes de la représentation de l'entreprise, avait pour point de départ mon travail sur la critique de l'économie politique que Marx développe dans Le Capital, et la découverte de l'inachèvement de ce monument. Dans son mode d'exposition, Marx obéissait à la démarche scientifique classique, partant de l'établissement des concepts les plus abstraits pour avancer dans son analyse vers des concepts plus concrets. Pour nourrir sa conceptualisation, il s'appuyait sur une critique des doctrines économiques importantes qui l'avait précédée en procédant à un clivage entre elles, d'une part les grands de l'économie classique (dont Adam Smith et Ricardo sont les plus importants) et d'autre part, les représentants de l'économie vulgaire.

\section{L'entreprise comme modalité de l'agir}

Ma maîtrise m'avait conduite à prendre tout particulièrement en considération la partie de cette critique des théories économiques qui s'y applique, qui n'est pas dévalorisant comme on pourrait l'entendre, mais qui concerne ceux des économistes qui prennent pour point de départ ce qui se passe dans la pratique entrepreneuriale et s'appuie sur cette fameuse loi de l'offre et de la demande dans la détermination des prix. L'exemple type, pour nous, est Jean-Baptiste Say. Au fur et à mesure que j'avançais dans la lecture du Capital, mais aussi des Théories sur la plus-value et des Fondements de la critique de l'économie politique qui sont des fragments rassemblés qui devaient faire suite dans la rédaction du capital, j'y cherchais de plus en plus désespérément ce que pouvait être la critique de l'économie dite "vulgaire ", celle précisément qui entrait dans le champ de l'entreprise et des activités entrepreneuriales. Là-dessus, Marx annonce à plusieurs reprises qu'il va s'y consacrer, mais il n'a pu le faire et son ouvrage est resté inachevé. Voilà qui m'ancrait dans l'idée que c'était le plus intéressant à poursuivre. C'est à l'occasion de ce travail de maitrise que je rencontrais pour la première fois, Cantillon, son Essai sur la nature du commerce en général, c'est-à-dire aussi, l'invention du concept d'entrepreneur. Pour l'aborder je m'appliquais à mettre en œuvre ce que pouvait être une approche 
critique, les outils intellectuels qui permettent de se tenir au plus près de l'acte de concevoir, comprendre, juger dans une œuvre, dans un texte comme dans le déroulement d'un acte projeté.

L'autre source de mon choix de travailler sur l'entreprise à l'époque moderne est ce qu'au cours d'une étude sur Machiavel et le machiavélisme, je découvrais l'importance du concept d'entreprise politique à l'époque moderne. Alors s'est imposée la question: comment le concept économique d'entreprise avait-il pu prendre corps dans le contexte de la tradition anti-machiavélienne si présente dans l'Europe moderne, tradition dans laquelle l'entreprise politique est la subversion des liens de l'État? Entreprise économique, entreprise politique, Cantillon, Machiavel : s'ouvrait un champ de recherche passionnant sur la représentation d'un mode d'action qui s'incarnait dans les grands pôles de l'action : la guerre, le commerce, la justice, la production.

Sur le sujet de l'apparition tardive du concept d'entreprise dans la littérature économique anglo-saxonne, j'ai écrit l'article "entrepreneur ", dans le Vocabulaire européen des philosophies de Barbara Cassin (2004); j'ai essayé d'approfondir cette question dans un article sur "Les figures de l'entrepreneur : histoire et typologie ", paru en 2003. Enfin, dans l'article « Entrepreneur » de l'Encyclopédie historique, publié par Christophe Charles et Daniel Roche en 2018, j'ai élargi mes investigations aux autres langues européennes en essayant de dégager comment elles mettent l'accent respectivement sur des enjeux et des caractéristiques différents, tous issus des termes latins que l'on traduit par " entrepreneur »: redemptor, contractor, susceptor, conductor. Autant dire que la question des modulations du sens apportées dans nos langues à cette profession qui se dénomme comme elle se définit, c'est-à-dire comme une modalité de l'agir, n'a cessé de m'intéresser.

Entrepreneur, Entreprise. Histoire d'une idée a davantage intéressé les économistes que les philosophes et les historiens... au point que ton premier laboratoire d'accueil au CNRS a été un laboratoire d'économie. Ces années 1980/1990, moment de ton entrée au CNRS, ont été des années essentielles intenses d'échanges autour des sciences et ingénieries nouvelles, je pense à la science des systèmes, avec Jean-Louis Le Moigne, le traducteur des Sciences de l'artificiel, d'Herbert Simon, et aux nouveaux domaines de l'ingénierie qui se développaient autour de l'informatique, ingénierie système, ingénierie de la conception, ingénierie de l'organisation. C'est dans ce creuset qu'a été conçu La gloire des ingénieurs, ton second ouvrage.

En effet, lorsque je me suis présentée une première fois au CNRS en 1983, en philosophie, la majorité du jury a jugé que ce n'était pas un sujet de leur ressort. J'y suis entrée l'année suivante, avec le soutien de Jean-Louis Le Moigne, qui m'avait contactée après la publication de mon livre sur l'entreprise. J'avais commencé à travailler avec lui en 1983 dans son laboratoire du GRASCE, le Groupe de Recherche en Analyse de Système et Calcul Économique créé par Bertrand Munier et lui-même. J'ai donc proposé un nouveau projet au CNRS et ce sont les économistes qui m'ont accueillie. Mais mon intérêt pour Herbert Simon était antérieur. J'avais lu et relu la traduction et présentation de Science des systèmes, science de l'artificiel peu avant la soutenance de ma thèse en 1980. Les travaux et réflexions autour de la science de l'ingénieur chère à Jean Louis Le Moigne -on ne connaissait alors que la science "pour» l'ingénieur s'insurgeait-il- m'intéressaient forcément, puisque mon souci constant était de me tenir en ce lieu où la théorie devient pratique et où la pratique se cherche des outils théoriques. 


\section{Sciences de la conception, pensée complexe, pensée d'action}

Le premier résultat écrit de mon travail, fut un rapport de recherche, réalisé au GRASCE, qui prenait pour point de départ une déclaration d'Herbert Simon sur la modélisation de la décision dans laquelle il affirmait qu'elle était toute nouvelle et en quelque sorte ne commençait qu'avec l'approche qu'il prônait. Ce que je me suis donné pour objectif de réfuter en m'appuyant sur la réduction en art de la fortification au début du XVII e siècle. C'était en 1985. Ceci dit, on ne peut pas dire que mon « creuset » s'est « déterminé » à partir de mes activités au GRASCE. En revanche, il a été pour moi très stimulant de travailler dans cet entourage où l'on réfléchissait sur la décision, sa modélisation, le projet, les approches procédurales, qui étaient déjà mes objets de réflexion lorsque je travaillais pour ma thèse mais où ils étaient abordés de toute autre manière.

Néanmoins, tu as contribué à l'édition en français, en 1987, de l'article d'Herbert Simon, «Style in Design » (" Les apaisements de l'alternative ou la théorie des styles de H.A. Simon »). En quoi était-il important de faire s'arrêter sur cet aspect moins connu de la pensée de Simon?

En effet, j'ai traduit ce petit texte inédit en français, que Jean-Louis Le Moigne a commenté, pour le $n^{\circ} 2 d^{\prime}$ 'Amphion. Mais, c'est surtout dans la postface de ce numéro, dans laquelle j'ai essayé de synthétiser les idées exprimées dans l'ouvrage sur le fonctionnalisme en architecture, que j'ai pu à nouveau poser la question : où faut-il se tenir pour saisir l'essentiel de ce qui se joue dans un texte, une pensée, une œuvre? J'ai tenté de comprendre ce qu'il en était dans les écrits et les positions théoriques de Simon. Ce qui m'a fait tourner autour de son texte et m'a donné envie de le traduire est (entre autres) la question : comment en architecture faire coïncider au mieux dans la conception d'un bâtiment, l'aménagement de l'espace intérieur et le style architectural de l'extérieur d'un bâtiment? Une forme de questionnement sur la notion d'interface pour moi assez floue et en même temps très excitante. J'ai toujours essayé de réfléchir sur les points d'inflexion, d'articulation de la réflexion, là où s'exercent doute et projection vers l'altérité.

Quels ont été les apports réciproques entre «pensée complexe » (Jean-Louis Le Moigne) et "pensée d'action»?

Je dois dire que malgré toutes mes sympathies envers les porteurs de la "pensée complexe " j'ai été assez rétive à accepter le chamboulement de la logique -comme armature intellectuelle de notre rapport au monde- que proposaient joyeusement ses partisans. J'avais l'impression déroutante que tout y était réduit au même niveau d'abstraction, que les notions même de théorie, de concept, de jugement, devenaient inopérantes. Comment alors avancer dans la pensée ? Je crois que ce qui a davantage structuré ma manière d'aborder les questions épistémologiques autour desquelles je n'ai cessé de revenir est le travail critique de K. Marx dans Le Capital, et avec aussi mes lectures de Hegel, la réinterprétation de la dialectique. Pour l'illustrer, j'avais en permanence sur mon bureau, lorsque je travaillais à ma thèse un extrait de la Phénoménologie de l'esprit : 


\begin{abstract}
«La chose en effet n'est pas épuisée dans son but, mais dans son actualisation ; le résultat non plus n'est pas le tout effectivement réel, il l'est seulement avec son devenir » (préface p.7)
\end{abstract}

Je pourrais dire que cette phrase, toujours devant mes yeux, est restée un guide qui m'évitait de dévier vers cette sorte de religion du concept qui oublie sa forme vive, le jugement, ce qui met en relation.

Ce qui me gênait aussi dans la pensée complexe, est qu'elle s'est revendiquée en France sur un mode clairement réactif, celui d'une critique sévère et qui se voulait dévastatrice, s'élevant contre une pensée « cartésienne » qui n'est que la caricature d'un cartésianisme dégradé, ou un « positivisme » qui ne méritait peut-être pas d'être voué aux Gémonies. Cette démarche qui se crée un repoussoir pour mieux s'affirmer m'a toujours exaspérée. Par ailleurs j'avais été particulièrement impressionnée par la thèse de Jean-Pierre Séris et les lumières qu'elle apportait sur les nouveaux rapports entre les savants et les techniques au début du XVIII ${ }^{e}$ siècle. C'est en me lançant dans l'étude historienne de cette période que j'ai commencé à fréquenter l'Institut d'histoire et de philosophie des sciences et des techniques (IHPST), et en particulier les séminaires de Jacques Guillerme, que j'ai rencontré en 1983. Je le connaissais par son article sur la technologie dans Thalès dont m'avaient parlé Etienne Balibar et Pierre Macherey -nous étions voisins. Avec ce dernier, dès 1985, nous nous sommes attelés à l'écriture d'articles en commun. Et, ensemble, nous avons organisé son séminaire qui s'est très vite centré sur l'ingénieur Raucourt de Charleville. Ce séminaire réunissait des chercheurs dont plusieurs se sont imposés ultérieurement comme Antoine Picon, Georges Ribeill, Georges Vigarello...

En parallèle, avec Jacques Guillerme, dans ces mêmes années, vous avez largement renouvelé l'approche conceptuelle du « projet » et de la « conception ». Peux-tu évoquer ces travaux dont l'impact a été considérable auprès des architectes et des chercheurs en gestion des connaissances? Quelle filiation y a-t-il avec la pensée de G. Canguilhem, qui dirigeait alors la revue Thalès?

Ce qui a été intellectuellement très fécond dans notre rencontre, c'est que nous venions de deux mondes très différents, lui, de la physico-chimie et de l'histoire de l'art -en particulier de l'architecture-, et moi de la philosophie et engagée dans des travaux historiques, mais que nous avions envie de creuser les mêmes questions, sur la technique, sur la technologie, la conception de projet, les aléas de l'idée d'innovation, et ferrailler contre l'idée d'application. Jacques Guillerme était déjà bien introduit dans le milieu des historiens de l'architecture, plus à l'étranger qu'en France. Nous avons écrit une petite dizaine d'articles ensemble entre 1985 et 1989, sur la normalisation, la standardisation, les essais d'optimisation techniques tels qu'ils se concevaient avant la production industrielle. Notre collaboration a aussi été l'occasion d'aborder de nouveaux domaines. Par exemple, j'apportais mon intérêt pour la gestion des matériels dans les arsenaux de marine, et Jacques Guillerme, ses réflexions sur les collections. Cette conjonction était propice à toutes sortes de renouvellement des questionnements sur l'histoire du projet de production, le choix de l'élément pertinent dans la résolution d'un problème technique. Nous avions aussi des discussions renouvelées pour ce qui constitue des sortes de champs intermédiaires dans la conceptualisation : les règles, les règlements, la norme et les standardisations, toute l'épaisseur des possibles et des contraintes qui sont proprement palliées -recouvertes comme par un manteau- à l'aide de la notion 
d'application, simple moyen de se soustraire à un véritable questionnement sur les rapports entre la théorisation propre aux ingénieurs et leurs pratiques effectives. Ceci dit, l'impertinence intellectuelle de Jacques Guillerme convenait tout à fait à mes impatiences vis-à-vis de l'institution, dans laquelle nous n'avons, ni l'un ni l'autre, fait carrière.

Quant à l'apport de Georges Canguilhem, il était très important pour Jacques Guillerme et je sais qu'il y avait beaucoup d'estime réciproque entre eux. Canguilhem est venu à plusieurs reprises assister à notre séminaire rue Dufour. Quand je lui ai envoyé mon livre sur l'entreprise, et que je lui ai avoué combien sa rigueur intellectuelle m'avait été un modèle (en particulier, sa manière de traiter l'histoire d'un concept), il s'est défendu d'une telle rigueur et a toujours été très encourageant. Grâce à Camille Limoges ${ }^{8}$ j'ai pu accéder à une meilleure appréhension de l'étendue de son apport à l'histoire des sciences et aussi des techniques.

Peux-tu nous parler d'Amphion ? En quoi ce projet de revue était-il différent de Thalès ?

La revue Thalès dont le premier tome daté de 1934 est publié en 1935, se présente comme un «Recueil annuel des travaux de l'Institut d'Histoire des sciences et des techniques ». Il a connu 12 tomes dont le dernier date de 1966 (publié en 1968). Le projet d'Amphion, Études d'Histoire des techniques était typiquement dans l'esprit du travail de Jacques Guillerme : rassembler les réflexions courtes et argumentées de spécialistes portant sur des textes historiquement pertinents sur le thème général choisi. Ce n'est pas par hasard si les deux premiers fascicules de cette publication ont porté sur le fonctionnalisme en architecture, qui était un thème de réflexion cher à $\mathrm{J}$. Guillerme et qui constituait l'un de mes axes de réflexion. À l'époque, je travaillais surtout sur l'administration de la marine, la construction navale et l'organisation des chantiers aux $\mathrm{XVII}^{\mathrm{e}}$ et $\mathrm{XVIII}^{\mathrm{e}}$ siècles où l'idée de fonction était omniprésente. Amphion a donné lieu à trois publications : en 1987, Le fonctionnalisme en architecture, en 1989, Nicolas-Claude Fabri de Pereisc, Lettres à Cassiano di Pozzo (1626-1637) et en 1993, Les collections. Fables et programmes.

C'est là, j'imagine, que La gloire de l'ingénieur s'est imposé comme second grand projet d'écriture.

Oui, et à l'occasion de mon article paru dans la revue Synthèse en avril-juin 1987 sur la construction en une journée d'une frégate à Toulon en 1679 j'ai parlé à Jean Claude Perrot avec enthousiasme de ce monde des chantiers que je découvrais et auquel j'avais envie de consacrer mon travail. Jean-Claude Perrot dirigeait alors cette revue et la collection «Évolution de l'humanité » chez Albin Michel, et il m'a demandé de proposer un synopsis. À vrai dire, l'idée était déjà évoquée dans mon projet de candidature au CNRS. C'est en travaillant sur les entrepreneurs à l'époque moderne que le personnage de l'ingénieur a commencé à m'intéresser. Mais encore une fois, ce qui m'importait d'abord était de comprendre en quoi la position très particulière des ingénieurs et la triple exigence à laquelle était soumis leur travail -économie, temps, efficacité technique- les a conduits à produire certaines formalisations de l'action qui les faisaient en particulier accéder à l'anticipation des « espérances conceues » comme ils le disent.

\section{De la relation entre pratique et technique}


Dans La gloire de l'ingénieur, tu fais cette observation, remarquable à mon sens (p.342) : « en quelque sorte, l'Europe technicienne fut d'abord raccommodeuse : n'est-ce pas une façon de dire que la maîtrise pratique des savoir-faire précède leur maîtrise technique? ». Et tu relies cette question de la relation entre maîtrise pratique et maîtrise technique des savoirfaire au devis. Ce thème du « devis » est une constante dans ton œuvre.

Je donnerai deux exemples de cette importance du raccommodage : le fait que dans la construction des machines telle qu'elle est décrite dans les théâtres de machines, pourtant soupçonnés de fantaisies mécaniques, faciliter le remplacement et l'entretien des pièces est l'une des anticipations qui orientent les choix des concepteurs, en particulier le plus prolifique d'entre eux, Ramelli. L'autre exemple est celui du navire, les Anglais étant les champions de la maintenance par raccommodages successifs qui leur faisait dire que c'était et ce n'était plus le même «Vieux Jacques » vieux de 25 ans, qui naviguait encore, toutes les parties ayant été changées.

Quant à la pratique précédant la technique, on peut d'abord remarquer que "réduire en art" s'annonce précisément comme faire passer de la pratique à l'art ou à la méthode, c'est-à-dire à une maîtrise de l'action qui implique d'autres facteurs ou d'autres procédés et surtout, offre d'autres libertés de choix. Par exemple, dans la construction, une maîtrise du dimensionnement, la possibilité de faire jouer le rapport dimensions et proportions afin de produire de nouveaux effets. Cela suppose que l'on dispose de la capacité de faire un usage différent de l'arithmétique et de la géométrie, alors même que l'on recourt à la géométrie pratique instrumentale. Celleci, aussi merveilleusement subtile qu'elle soit, ne permet pas que l'on se délivre de la succession stricte des gestes opératoires traditionnellement répétés dont l'un dépend de l'autre, selon la parole apprise du maître : fais ceci, puis cela, puis cela, etc., et tu obtiendras par exemple la spirale que tu recherches. On pourrait même dire que lorsqu'on passe de la pratique à l'art, l'usage de l'instrument se retourne, il ne se présente plus tant comme le jeu des proportions données selon certaines grandeurs prédéfinies, que comme l'organe qui définit des proportions réalisables dans des grandeurs potentiellement utiles. Ainsi du compas de proportion : c'est la conception mise en œuvre dans son usage qui peut changer. On pourrait en conclure qu'il est bien difficile de faire une stricte ligne de démarcation entre pratique et technique, car dans les faits elles interfèrent.

Il y a deux façons, il me semble, de considérer la question de la successivité : maîtrise pratique puis maîtrise technique. La première considère ce phénomène dans le temps et l'espace historique, ce que souligne la référence à l'Europe technicienne. Cela vaut sans doute en gros mais pas au détail. La deuxième façon aurait plutôt trait aux modifications des procédés productifs eux-mêmes. On peut y considérer deux cas possibles : soit la maîtrise pratique se présente comme une contrainte que l'approche proprement technique doit prendre en compte, ainsi de la conception de l'ingénieur face aux savoirs de ses ouvriers sur lesquels il s'appuie et sans lesquels il ne peut rien; soit la maîtrise pratique peut devenir un handicap, l'approche technique exigeant un renouvellement complet non seulement des actions à produire mais de l'ordre à tenir dans l'ensemble des actes nécessaires pour obtenir les effets escomptés.

Quant au devis, il répond à deux sortes d'impératifs: rendre raison de/par l'évaluation technique et rendre compte de/par l'estimation monétaire des travaux, 
que les choix proposés sont les meilleurs possibles. Or l'évaluation technique et l'estimation monétaire qui doivent coïncider terme à terme lorsque l'on dresse le devis, répondent à des logiques opératoires complètement différentes. Les faire coïncider terme à terme est procéder à des réductions qui s'opèrent toujours dans le même sens : réduire des facteurs hétérogènes en grandeurs calculables en termes monétaires. Autrement dit, la recherche d'une optimisation technique implique une combinatoire entre l'espace, le temps, les choses, les hommes, qui peut se décliner diversement alors que l'estimation monétaire traite ces facteurs comme des marchandises ayant un prix de marché. Le devis devient ainsi le document qui formalise des impératifs sociaux, politiques, économiques, dans cette mise en parallèle de l'évaluation technique et de l'estimation monétaire et constitue de ce fait ce que j'ai appelé un document technographique.

\section{Technologie : la fortune du mot}

Si je fais un pas de côté (encore que...), j'observe qu'un autre thème court dans toute ton œuvre, celui de la technologie. Tu conclus La Gloire par un chapitre court et lumineux, que tu intitules "l'inachevable technologie». Et tu termines ainsi : "Le principe d'ordre des premières classifications des arts fut très vite d'être potentiel », du simple fait que l'art luimême (au sens que lui donnaient les modernes) est potentiel : l'ingénieur talentueux n'est-il pas celui qui anticipe, bien qu'il ne sache pas, au juste, ce qui va advenir. Anticiper malgré, n'est pas tout prévoir, mais se disposer à l'imprévu... ». Propos justes et profonds, qui résonnent bien avec les théories actuelles de la conception innovante développées par Armand Hatchuel, Benoît Weil et Pascal Le Masson, au Centre de Gestion Scientifique de Mines ParisTech. Néanmoins, Réduire en art porte en sous-titre: " la technologie de la Renaissance aux Lumières », et dans un article passionnant, tu parles de la "technologie, science intermédiaire »? Sommes-nous décidément face à une technologie introuvable, comme l'écrivait Jean-Claude Beaune?

L'histoire de ce mot «technologie » exige surtout et avant tout que l'on ne cède pas à la tentation d'y trouver un sens même pas un sens "univoque" mais plus basiquement encore des sens compatibles entre eux. Car, qu'y a-t-il de commun entre le «technologue » d'Aristote, qui est le rhéteur qui ne craint pas de convaincre le juge en jouant sur ses émotions, le «technologue » selon Saint Basile, qui trompe le chrétien en dénonçant la présence du Christ dans le pain consacré, ou alors la filiation du Christ Dieu comme incompatible avec l'éternité divine, la «technologie » comme l'ordre à tenir entre les livres d'une bibliothèque, la «fausse science des mots ", sens encore en usage au début du $\mathrm{XX}^{\mathrm{e}}$ siècle, cette sorte de malversation de la rhétorique qui, il est vrai, nous ramène à Aristote? Jacques Guillerme a soigneusement réuni une quantité incroyable d'usages et de définitions de ce mot qui avaient commencé à être rassemblés lors du fameux séminaire à l'IHPST de 1963-64 et 64-65, d'où est sorti l'article sur « Les commencements de la technologie » et aussi les définitions, dont quelques-unes apparemment plus ou moins aberrantes, publiées à la fin du même recueil par J. Morère.

Dans l'histoire de ce terme, la première référence aux abus de la rhétorique ou à l'art du classement des livres, est cependant bientôt écartée par l'intérêt manifeste et nouveau de la Renaissance pour l'action face à la contemplation, jusqu'alors seule digne de reconnaissance. Le principe selon lequel dorénavant on considèrerait que la contemplation sans l'action n'était qu'une activité humaine amputée en quelque sorte de son aboutissement, de son achèvement, est un renversement frontal sans 
lequel l'importance de la réduction en art ne saurait s'apprécier ni d'ailleurs se concevoir. Au XIX siècle, l'essor des encyclopédies, manuels ou revues qui se réclament de la technologie a tout à voir avec l'essor industriel. Cependant on a beaucoup glosé sur le « retard» français à l'adoption du terme, et, avec lui pensaiton, sur une faiblesse. Il faudrait plutôt considérer que la fortune du terme dans les pays protestants - et même anglicans ou puritains - tient à sa présence insistante dans les titres mêmes de traités théologico-philosophiques où s'affrontent différentes applications du terme en compétition. Christian Wolff décide que le domaine de son application est le monde des arts mécaniques, alors que d'autres parmi ses prédécesseurs l'affirmaient uniquement utilisable dans le domaine des arts libéraux. Sans doute, c'est Christian Wolff qui a retenu l'attention des encyclopédistes français.

\section{Jalons pour une histoire de la confiance}

Peux-tu nous parler de L'Inscription sociale du marché, que tu as publié avec Annie Jacob ? Un ouvrage trop méconnu à mon sens...

Le titre de cet ouvrage, L'inscription sociale du marché, correspond au thème général du premier séminaire de l'ADSE, association pour le développement de la socioéconomie, branche française, de la SASE (Society for the Advancement of SocioEconomics), fondée en 1990 et qui existe toujours. Notre association lancée par Romain Laufer, et à laquelle je me suis immédiatement associée, a très vite réuni de nombreux chercheurs. Le thème «Inscription sociale » renvoie à la notion qui nous intéressait : « embeddedness » (Mark Granovetter lui consacre un article de l'ouvrage), et l'objectif de notre association était, comme celle de la SASE, de sortir l'économie politique de son réduit mathématisant et de confronter ses concepts et ses conceptualisations aux différentes sciences sociales. Plus généralement, il s'agissait de promouvoir la pluridisciplinarité dans le domaine des sciences sociales. Les travaux de nos séminaires passionnants et passionnés se sont tenus régulièrement à Paris jusqu'en 1999. Ils réunissaient des historiens, des juristes, des sociologues, des économistes, des philosophes, des anthropologues, qui ont depuis fait leur chemin. Et cela a donné lieu à la publication de trois colloques : L'inscription sociale du marché, 1995 (Lyon 1992), La confiance en question, 2000 (Aix-en-Provence 1996), La production des normes entre État et société civile: Les figures de l'institution et de la norme entre États et sociétés civiles, 2000 (Villeneuve d'Ascq, 1997).

La confiance, précisément: c'est le thème de ce remarquable article, trop peu connu là encore: "Jalons pour une histoire de la confiance », dans lequel tu proposais des raisons et des axes de travail... pour aborder historiquement un sujet devenu majeur, c'est le moins que l'on puisse dire...

Le thème de la confiance avait fait l'objet de notre séminaire de l'ADSE en 1995, auquel avait contribué des membres du Mauss dont la revue ${ }^{\circ} 4$ de 1994 «à qui se fier? Confiance, interaction et théorie des jeux », avait retenu mon attention et c'est en prenant pour point de départ des remarques et analyses d'Alain Caillé et de Jacques T. Godbout, que j'ai tenté de construire une sorte de grille de lecture ou de schéma propre à faire de " la question de la confiance » un objet d'étude alors qu'elle se présente toujours sous une forme aporétique. Mon hypothèse est qu'on ne peut dépasser l'aporie et donc "formuler la question » que si l'on reconnaît qu'elle est constitutive de l'individu entendu comme une personne, dans l'épaisseur contradictoire de la conscience de soi confrontée au monde environnant. Ce que j'ai 
essayé de développer à l'aide de diverses solutions mises en œuvre dans notre tradition.

La première et la plus immédiate qui me soit apparue est exhibée dans cet étrange rapport entre le passager du métro et le mendiant en ce que celui-ci démontre et réclame sa qualité de personne; elle est ritualisée, expulsée dans un autre espace avec le bouc émissaire; elle se résout dans la vie est belle de Franz Cappra en rappelant que nous avons plusieurs vies, qu'elles peuvent être hétérogènes et en même temps constituer un tout assignable ; elle se révèle dans le roman de Chrétien de Troyes dans l'impossibilité de Perceval à poser la question qui lui permettrait de voir le Graal. Si la condition de possibilité de la question de la confiance est constitutive de l'individu, alors les Exercices spirituels d'Ignace de Loyola en sont la preuve a contrario, en exacerbant l'état d'aporie, la suspension du jugement, jusqu'au perinde ac cadaver-disponible comme un cadavre; et enfin Montaigne en ses Essais ne s'y dérobe pas : le divisé, l'inconciliable est présent en soi et de soi à soi et c'est vrai aussi « en notre bâtiment public ». A plus d'un égard on peut dire que l'impossibilité de trouver le socle où poser la question de la confiance fait la matière des Essais.

\section{Autour de la philosophie de l'action}

Dans les années 2000, avec Romain Laufer (HEC) et Armand Hatchuel (CGS, Mines Paris Tech), vous avez animé un séminaire au Collège International de Philosophie, qui lui aussi a fait date autour des notions d'entreprise, de construction des énoncés techniques, du travail comme valeur philosophique. J'ai souvenir de débats passionnants... et de contributions majeures à l'interprétation de l'évolution intellectuelle du monde des années 2000-2010.

Le séminaire du Ciph a commencé en 1991 sous l'impulsion de Romain Laufer (HEC) et à son commencement, l'appui de Maria Bonnafous-Boucher (HEC). Nous l'avons continué à quatre, avec Romain Laufer, Armand Hatchuel (CGS Mines), Jean-François Bordron (sémiologie Paris III) et moi-même. Pierre Guillet de Montoux qui nous apportait ses réflexions sur les rapports de l'art et du marché s'est régulièrement joint à nous, et Anne-Françoise Garçon, la dernière année. Le thème général de ce séminaire était «Philosophie, entreprise et management, les nouveaux lieux de la philosophie de l'action ». J'y trouvais encore une fois l'interdisciplinarité, si vantée au CNRS et si bien empêchée. Le séminaire s'est tenu au collège de philosophie à partir de 1995 et a donné lieu à plusieurs colloques. Deux d'entre eux ont été publiés, R. Laufer, A. Hatchuel, Le libéralisme, L'innovation et la question des limites (2005) et Ruth Bereson, Pierre Guillet de Monthoux, Pragmatism, Art and Management (2017). Des colloques organisés à Cerisy par Armand Hatchuel, sur la rationalité, sur la conception, sur le marché, ont été l'occasion de poursuivre notre réflexion.

Qu'entendiez-vous par «philosophie de l'action »? Et : n'était-ce pas une manière autre de renouer avec Entrepreneurs, entreprise... ?

Certainement pour moi. En fait, nous n'avons pas tenté de produire une approche commune, mais plutôt de réfléchir à plusieurs mains, si j'ose dire, à des questions qui avaient quelque rapport avec le management, l'action, non sans parfois se donner quelque licence sur l'extension du thème. C'est ainsi que j'ai énormément travaillé pour l'un de nos séminaires qui portait sur la notion de valeur, sur L'Esprit des Lois et les Pensées de Montesquieu. Ceci, parce qu'en travaillant sur l'entreprise de guerre et la réduction en art de l'attaque des places, j'avais découvert le grand clivage que 
Montesquieu déploie entre l'honneur, valeur noble et le calcul, estimation mercantile qui atteint le domaine de la guerre par les estimations des ingénieurs.

\section{« Réduire en Art » : genèse d'un ouvrage}

Les années 2010 ont été consacrées à "Réduire en art », une recherche que tu as voulu collective, pour ne pas dire pluridisciplinaire, avec un séminaire au Centre Alexandre-Koyré, et un ouvrage collectif, que tu as publié avec Pascal Dubourg-Glatigny. Et là encore, j'ai souvenir d'échanges passionnants, sur un sujet qu'à la fois nous découvrions collectivement, dont nous prenions la mesure, l'ampleur historique. Ce mouvement de pensée a structuré les énoncés techniques européens, durant près de deux siècles. C'est pour toi l'un des moments fondateurs de la pensée technologique occidentale...

C'est dès 1985, à l'occasion d'un rapport de fin de contrat avec Jean-Louis Le Moigne que j'ai pour la première fois découvert l'expression "réduire en art » et son importance dans la formalisation de l'activité technique au tout début du XVII ${ }^{\mathrm{e}}$ siècle. Ce qui m'apparaissait déterminant alors était le travail de modélisation de la décision que cette « réduction » permettait. C'était dans le domaine de la fortification, avec La fortification réduite en art de Jean Errard. J'ai découvert progressivement l'ampleur de ce phénomène de réduction en art dans d'autres domaines: architecture, charpenterie, construction navale, et puis bientôt s'appliquant à l'ensemble des activités techniques, y compris des arts comme la danse, l'escrime mais aussi à des activités intellectuelles comme la conception de l'histoire et les remaniements du droit. Deux autres axes se sont alors présentés qui encore élargissaient l'étendue du domaine considéré. Dans le temps, la découverte de l'origine latine et surtout cicéronnienne de l'expression et de son extension, sous la plume de Cicéron, à partir du domaine juridique vers les autre activités productives et scientifiques, et durant le $\mathrm{I}^{\text {er }}$ siècle avec Varron, s'agissant du rangement des livres, de leur conception matérielle, et de leur organisation interne, avec Frontin pour l'organisation de la conduite des eaux, et bien sûr avec Végèce pour les arts de la guerre, avec Vitruve pour l'architecture et la mécanique, pour la production d'une géométrie pratique détachée des recettes artisanales, et délivrant un nouveau champ conceptuel et décisionnel. J'ai consacré plusieurs articles à ce sujet au fur et à mesure de la découverte de son extension dans le temps et dans ses domaines d'application. L'autre axe qui s'ouvrait à ma recherche à partir de mes investigations sur la réduction en art, était la question de la méthode, - on disait réduire en art ou en méthode - l'importance de cette question au $\mathrm{XVI}^{\mathrm{e}}$ siècle et donc aussi une relecture du Discours de la méthode de Descartes à partir de ces réductions en art et méthode du $\mathrm{XVI}^{\mathrm{e}}$ siècle.

En effet, très vite, parce que je décidais de travailler sur ce qu'étaient alors les ingénieurs, je m'étais attachée à comprendre l'histoire de ce mot : ingénieur et dès 1984, j'ai écrit un article « le mot ingénieur » qui a fait son petit chemin et a été bien utilisé. Bien sûr ce travail s'est poursuivi et j'ai été amenée à réfléchir sur la notion d'ingenium. La littérature sur ce thème est au $X I^{e}$ siècle assez pléthorique, en particulier dans la littérature italienne et en un sens non négligeable, représente l'un des volets d'un couple : exercer son ingenium / réduire en art et méthode, et plus succinctement ingenium/méthode, pouvoir d'inventer propre à ceux qui sont doués d'ingenium d'une part et moyen de donner à tous la méthode de procéder sûrement dans la pensée comme dans l'action, d'autre part. À partir de là bien des questions se 
posaient et en particulier, faut-il lire les Regulae ad directionem ingenii - inachevées et le Discours de la méthode comme la forme cartésienne des interrogations propres à son temps concernant ces deux démarches?

En 2002, j'ai décidé que je consacrerai les dernières années de mon activité au CNRS à un travail sur « réduire en art » et surtout, je voulais que ce soit un travail collectif. J'ai donc repris la direction du séminaire organisé au centre Koyré, «Formation des savoirs à l'époque moderne », en l'axant plus précisément sur ce sujet : « réduire en art », la technologie de la Renaissance aux Lumières. J'ai demandé à Pascal Dubourg Glatigny, chercheur en histoire de l'art au centre Koyré, de s'y associer et nous avons lancé un appel pour trouver des partenaires dans ce travail d'exploration. Nous avons formé ainsi un groupe de chercheurs qui s'est maintenu pendant les deux années suivantes.

Notre méthode était la suivante : on présente un exposé oral. En tenant compte des remarques et critiques des autres membres du séminaire, on produit un texte. $\mathrm{Ce}$ texte est soumis à la lecture de tous, et au cours d'une séance ultérieure, il est soumis aux remarques critiques issues de ces lectures et donne lieu à une réécriture. Ainsi chaque texte est passé par deux fois à la moulinette critique. Mais l'essentiel de ce qui était recherché par cette démarche et qui ressortait de nos discussions souvent passionnées, a été pour chacun d'entre nous l'occasion d'approfondir et d'enrichir sa compréhension de son objet d'étude propre, dans le contexte plus large où il se déployait. Pour moi, c'était une sorte d'expérience des jeux complexes entre concret et abstrait, une tentative pour faire vivre ensemble un processus d'abstraction et faire émerger un concept.

\section{Léonard de Vinci, Antoine Raucourt et la méthode}

Cet interview va être publié dans un numéro d'e-Phaistos intitulé "Autour de Léonard», dans lequel nos auteurs ont réfléchi à l'apport du grand ingénieur-artiste. Toi-même, au-delà du mythe fondateur et de l'engouement engendré par ses carnets d'ingénieur, comment situes-tu l'apport de Léonard de Vinci dans l'histoire de la pensée technique occidentale?

À part le Trattato della pittura publié en 1651 par Rafaelle Dufresne, l'œuvre de Léonard, est restée inédite jusqu'au XIX ${ }^{\mathrm{e}}$ siècle dans sa seconde moitié. C'est alors que l'on commence à s'intéresser d'abord, à Léonard savant, puis, ingénieur. J'ai travaillé sur cette apparition tardive et pour comprendre sa raison - ses raisons d'être alors. Il est certain que la capacité de reproduire en quantité les manuscrits de Léonard a été l'un des facteurs de ce mouvement de curiosité et de reconnaissance, amplifié considérablement par l'essor d'innovations techniques contemporaines comme l'avion, la bicyclette, le char, sujets déjà abordé dans son temps " précurseur » par Léonard. À partir des années 1880, Léonard de Vinci devient l'objet d'étude de grands esprits, comme Valéry. Si l'on ne souscrit pas à la méchanceté délicieuse de Cioran qui prétend que Valéry ne parlait jamais que de lui-même, on peut reconnaitre dans son essai qu'il a touché à la question toujours renouvelée de sa méthode et en particulier, comment Léonard associait des expériences sensibles patiemment renouvelées avec les fulgurances de ses analogies.

Enfin, sur quoi travailles-tu actuellement?

J'ai entrepris de venir à bout d'un ouvrage consacré à l'ingénieur Antoine Raucourt (1789-1841). Raucourt avait fait l'objet d'un séminaire de Jacques Guillerme à partir 
de 1986 auquel j'avais activement contribué en m'attachant aux recherches en archives et à leur exploitation. Je m'en étais éloignée pour travailler à mon livre sur les ingénieurs. A I'IHPST le projet d'une publication sur Raucourt avait été poursuivi par Jacques Guillerme puis interrompu par la maladie. Sans revenir sur les péripéties de ce projet je dirais que je l'ai repris à cause de l'intérêt que présente ce personnage, polytechnicien et ingénieur des ponts-et-chaussées, en ce qu'il s'est trouvé, au cours de sa vie, contribuer à des innovations techniques, organisationnelles, à des propositions de réforme de l'administration pénitentiaire, et à une sorte de réforme de l'individu grâce à l'enseignement d'une Philosophie positive dont il est l'auteur et le propagateur philanthropique dans des cours aux ouvriers.

C'est un cas intéressant en ce que ces innovations sont interconnectées. Il en tire un modèle d'organisation qu'il va renouveler en Russie, dans les ports de la mer Noire, et réinterpréter en fonction d'une situation bien différente du point de vue des conditions de l'appareil productif et des pouvoirs des administrations concernées. La dernière partie de sa vie est pour l'essentiel consacrée à des activités philanthropiques. Elles s'inscrivent dans des courants de pensées contemporains où il se réclame de la philosophie positive et défend, en héritier de Cabanis, une approche matérialiste de la morale, dans les courants pédagogiques alors très vivaces avec ses cours aux ouvriers, d'abord dans l'association polytechnique dont il est rapidement exclu, dans le cadre des réformes socio-politiques par ses relations et sa rupture avec les saint-simoniens, dans le cadre de l'académie des sciences et aussi du Génie civil, journal dont les colonnes lui sont ouvertes. Il est là où les idées et les choses bougent, y compris, comme on peut le voir dans le journal qu'il fonde, l'Éducateur, dans l'intérêt qu'il porte aux difficultés non seulement matérielles et physiques, mais aussi sociales et morales que rencontrent les classes défavorisées et en particulier les femmes.

La forte cohérence de son approche, sur tous ces fronts, tient à un souci constamment revendiqué, celui de se tenir en ce point de rencontre entre les savoirs de ceux qui sont impliqués ensemble dans un même projet: savoirs scientifiques, savoirs ouvriers sur les chantiers, mais aussi positivisme et sciences de l'homme dans la société avec une question récurrente : comment faire en sorte que l'échange des informations et la coordination des actes s'organisent au plus près et avec le maximum d'avantages.

Voilà un ouvrage que nous lirons avec plaisir ! Merci chère Hélène, au nom de l'équipe d'ePhaistos et des lecteurs, d'avoir accepté de nous consacrer cet interview. Et donc, nous attendons ton « Raucourt » avec impatience...

Ce sera sans doute pour l'année prochaine...

\section{BIBLIOGRAPHIE}

Sont retenus ici les ouvrages et articles évoqués dans l'interview 
GRANOVETTER Mark, « La notion d'embeddedness », in JACOB Annie, VÉRIN Hélène (dir.), L'inscription sociale du marché, Paris, L'Harmattan, 1995, p.9-19

GUILLERME Jacques, Sebestik Jan, « Les commencements de la technologie », Thalès, vol.12, 1966, p.1-72 ; URL : https://journals.openedition.org/dht/1226?file=1

GUILLERME Jacques (dir.), Amphion. Études d'histoire des techniques, t.I, Le droguier du fonctionnalisme; t.II: L'officine du fonctionnalisme, Paris, Picard, 1987

GUILLERME Jacques (dir.), Amphion, Études d'histoire des techniques 3. Les Collections : fables et programmes, Seyssel, Éditions Champ Vallon, 1993

GUILLERME Jacques, VÉRIN Hélène, « The Archeology of Section », Perspecta 25. The Yale Architectural Journal, 1989, p.226-257

CARVAIS Robert, GARÇON Anne-Françoise et al. (dir.) Penser la technique autrement : XVI ${ }^{e}-X X{ }^{e}$ siècle. En hommage à l'œuvre d'Hélène Vérin, Paris, Classiques Garnier, 2017

DUBOURG GLATIGNY Pascal, VÉRIN Hélène, Réduire en art : la technologie de la Renaissance aux Lumières, Paris, Éditions de la MSH, 2008

HATCHUEL Armand, WEIL Benoît, Les nouveaux régimes de conception. Langages, théories, métiers, Paris, Hermann éditeurs ( 2 ème édition), 2014

JACOB Annie, VÉRIN Hélène (dir.), L'inscription sociale du marché, Actes du colloque de l'Association pour le développement de la socio-économie, Lyon, novembre 1992, Paris, Éditions L'Harmattan, 1995

LAUFER Romain, ORILLARD Magali (dir.), La confiance en question, Paris, Éditions L'Harmattan, 2000

SÉRIS Jean-Pierre, Machine et communication. Du théâtre des machines à la mécanique industrielle, Paris, Librairie Philosophique J. Vrin, 1987

SERVERIN Évelyne et BERTHOUD Arnaud (dir.), La production des normes entre état et société civile : les figures de l'institution et de la norme entre états et sociétés civiles, Actes du troisième colloque de l'Association pour le développement de la socio-économie, Villeneuve d'Ascq, décembre 1997, Paris, Éditions L'Harmattan, 2000

SIMON Herbert A., « Du style dans la conception », traduction H. Vérin, suivi d'une « Présentation » rédigée par Jean-Louis Le Moigne et d'une Apostille spécialement rédigée par Herbert A. Simon en 1986, Amphion, Études d'histoire des techniques, vol 2, 1987 ; URL : http:// www.intelligence-complexite.org/fileadmin/docs/lesintrouvables/simon4.pdf

SIMON Herbert A., Les sciences de l'artificiel, présenté et traduit par Jean-Louis Le Moigne, Paris, Gallimard, 2004

VALERY Paul, Introduction à la méthode de Léonard de Vinci, Paris, Gallimard, (1894) 1992

VÉRIN, Hélène, Entrepreneurs, entreprise. Histoire d'une idée, Paris, PUF, 1982, rééd. Paris, Classiques Garnier, coll. « Histoire des techniques », 2011, Coll. poche : 2018

VÉRIN, Hélène, « Le mot : ingénieur », Culture technique, 1984

VÉRIN Hélène, « Un document technographique : le devis. Marine Royale et fortifications aux $\mathrm{XVII}^{\mathrm{e}}$ et XVIII ${ }^{\mathrm{e}}$ siècles ", Techniques \& culture, 1987, n 9, p.141-167; DOI : 10.4000/tc.868

VÉRIN Hélène, « Spectacle ou expérimentation La préfabrication de vaisseaux en 1679 », Revue de synthèse, $n^{\circ} 2$, 1987, p.199-223 ; DOI : 10.1007/BF03189055 
VÉRIN Hélène, «Évaluation des avantages du recours à l'entreprise dans les grands travaux de l'État (XVIII) », Politiques et management public, vol.7, 1989, p.57-77 ; DOI : 10.3406/pomap. 1989.2867

VÉRIN Hélène, La gloire des ingénieurs. L'intelligence technique du XVI $I^{e}$ au XVIII $I^{e}$ siècle, Paris, Albin Michel, 1993

VÉRIN Hélène, « Georges Canguilhem et le génie », in Georges Canguilhem, historien des sciences, Actes du colloque, 6-7-8 décembre 1990, Paris Albin Michel, 1993, p.76-89

VÉRIN Hélène, «L'État et l'entreprise au XVIII siècle : la gestion des ressources », Actes du colloque : Acteurs privés, acteurs publics : une histoire du partage des rôles, 8-9 nov. 1993, Techniques, Territoires et Sociétés, $n^{\circ} 27,1994$, p.89-95

VÉRIN Hélène, « Des propositions de gestion pour les arsenaux », Gérer \& Comprendre, Annales des Mines, n42, 1996, p.36-47; url : http://annales.org/gc/1996/gc_42_02_96.html

VÉRIN Hélène, « La réduction en art et la science pratique au XVI ${ }^{\mathrm{e}}$ siècle », Raisons pratiques, $\mathrm{n}^{\circ} 9$, 1998, p.119-144 ; DOI : 10.4000/books.editionsehess.10561

VÉRIN Hélène, « Jalons pour une histoire de la confiance », in LAUFER Romain, ORILLARD Magali (dir.), La confiance en question, Paris, L'Harmattan, 2000, p.37-57

VÉRIN Hélène, « Généalogie de la 'réduction en art'. Aux sources de la rationalité moderne », in GAUDIN Thierry, HATCHUEL Armand (dir.), Les nouvelles raisons du savoir : vers une prospective de la connaissance, Actes du colloque de Cerisy 2001, Avigon, éd. de l'Aube, 2002, p.29-41

VÉRIN Hélène, « Les figures de l'entrepreneur : histoire et typologie », in HATCHUEL Armand, LAUFER Romain (dir.), Le libéralisme, l'innovation et la question des limites, Éditions L'Harmattan, 2003, p.17-48

VÉRIN Hélène, « Entrepreneur », in CASSIN Barbara, Vocabulaire européen des philosophies, Paris, Le Seuil, 2004, p.353-357

VÉRIN Hélène, DOLZA Luisa, «Figurer la mécanique. Les théâtres de machines de la Renaissance ", Revue d'histoire moderne \& contemporaine, vol.51-2, n², 2004, p.7-37 ; DOI : 10.3917/ rhmc.512.0007

VÉRIN Hélène, «La pensée politique d'un ingénieur : l'exemple de la Vita Politica de Simon Stevin ", in SECRÉTAN Catherine (dir), Simon Stevin, De la vie civile, Lyon, ENS éd., 2005, p.109-129

VÉRIN Hélène, « La technologie : science autonome ou science intermédiaire? », Documents pour l'histoire des techniques, 2007, n¹4, p.134-143 ; DOI : 10.4000/dht.1210

VÉRIN Hélène, “'Comme en un infini' : les pouvoirs de la conception à la Renaissance », in HATCHUEL Armand, WEIL Benoît (dir.), Les nouveaux régimes de la conception. Langages, théories, métiers, Paris, Vuibert, 2008, p.17-38

VÉRIN Hélène, « Réduire en art », in GARRIC Jean-Philippe, NÈGRE Valérie, THOMINE-BERRADA Alice (dir.), La construction savante. Les avatars de la littérature technique, Paris, Picard, 2008, p.23-32 VÉRIN Hélène, « Les conditions de la rationalité technique des ingénieurs au temps de Vauban », in MARTIN Thierry et VIROL Michèle (dir.), Vauban, architecte de la modernité ?, P.U. de FrancheComté, Besançon, 2008, p.73-85

VÉRIN Hélène, « Marché et échange marchand : quelques jalons dans l'histoire d'un soupçon », in HATCHUEL Armand, FAVEREAU Olivier, AGGERI Franck (dir.), L'activité marchande sans le marché ?, Paris, Presses des Mines, 2010, p.65-75 ; DOI :10.4000/books.pressesmines.1228 
VÉRIN, Hélène, « Entrepreneur », in CHARLES Christophe, ROCHE Daniel (dir.), L'Europe.

Encyclopédie historique, Paris, Actes Sud 2018, p.550-553

\section{NOTES}

1. Johann Wolfgang von Goethe, Faust, trad. par Gérard de Nerval, Paris, Garnier Frères, 1877, p. 62 (Gallica.bnf.fr).

2. Y. Belaval (1908-1988), philosophe, grand spécialiste de Leibniz et du XVIII ${ }^{\mathrm{e}}$ siècle. Il fut attentif à ce qui unit les idées et les techniques.

3. Pierre Macherey était alors maître assistant à la Sorbonne. Philosophe, spécialiste de Spinoza, il s'intéresse aux approches littéraires de la pensée.

4. Pierre Vilar (1906-2003), historien de l'économie du XVIII ${ }^{\mathrm{e}}$ siècle en Catalogne, spécialiste des systèmes monétaires successifs (Or et monnaies dans l'histoire 1450-1920). Il a développé une réflexion approfondie sur le marxisme et la méthode de l'histoire dans son Histoire en construction. 5. Jean Toussaint Desanti (1914-2002), épistémologue, philosophe des mathématiques et phénoménologue a été un grand personnage de la philosophie du $\mathrm{XX}^{\mathrm{e}}$ siècle.

6. François Châtelet (1925-1985), philosophe, historien de la philosophie, co-fondateur avec Michel Foucault et Gille Deleuze, de l'université de Vincennes.

7. Jean-Marie Vincent (1934-2004), philosophe, chercheur en sciences politiques et militant cofondateur du PSU. Il a développé une approche originale de la critique de l'économie politique de Marx et de la littérature marxiste.

8. Camille Limoges a partagé son action et sa réflexion entre la promotion des sciences et des technologies à l'Université et au sein du gouvernement du Québec où il fut novateur et occupa de hautes fonctions. Élève de Canguilhem, il a contribué à la publication de ses œuvres complètes.

\section{INDEX}

Mots-clés : histoire des techniques, ingénieur, entreprise, pensée technique, technologie, énoncé technique, devis

Thèmes : Entretiens et leçons

Keywords : history of technology, engineer, technology, technical writing, technical design

\section{AUTEUR}

\section{HÉLÈNE VÉRIN}

Docteur d'État en philosophie, chercheur au CNRS en économie, philosophie puis histoire, ses recherches, centrées sur l'époque moderne, portent sur l'émergence et les transformations de l'idée d'entreprise, sur les premiers essais de formalisation des outils de décision par les ingénieurs, sur le vaste mouvement de « réduction en art et méthodes » des pratiques artisanales en Europe du XVI ${ }^{\mathrm{e}}$ au XVIII ${ }^{\mathrm{e}}$ siècle. Elle a publié Entrepreneurs, entreprise. Histoire d'une idée, Paris PUF 1982, réédité aux Éditions Classiques Garnier en 2011 et 2018 (collection Poche) ; La gloire des ingénieurs. L'intelligence technique du XVI au XVIII siècle, Paris Gallimard, 1993 (numérisé en 2009). 
Elle a dirigé l'ouvrage collectif Réduire en art. La technologie de la Renaissance aux Lumières, Paris, MSH 2008. Elle a consacré plusieurs articles aux Théâtres de machines. 